\title{
Impulsivity Components Measured by the Brazilian Version of the Barratt Impulsiveness Scale (BIS-11)
}

\author{
Componentes da Impulsividade Avaliados pela Versão Brasileira \\ da Barratt Impulsiveness Scale (BIS-11)
}

\author{
Alina Gomide Vasconcelos*, Maycoln Leôni Martins Teodoro, \\ Leandro Malloy-Diniz \& Humberto Correa \\ Universidade Federal de Minas Gerais, Belo Horizonte, MG, Brasil
}

\begin{abstract}
The main purpose of this study was to investigate the psychometric properties of the Barratt Impulsiveness Scale (BIS-11). Content item analysis was conducted by seven doctoral students. A convenience sample of 897 students was submitted to BIS-11 and they also filled self-reports about Minor Mental Disorder and ADHD symptoms, alcohol use and cigarette smoking. Mean age was 27.32 $(S D=8.69)$ years, $56 \%$ were female and $52 \%$ had incomplete college degree. Content and factorial analyses revealed that impulsivity was best represented by two latent factors labeled non-planning and inhibition behaviors. Test retest agreement tended to produce similar score patterns seven months after the first evaluation. Additionally, BIS-11 scores discriminated subjects in terms of cigarette smoking and psychopathological symptoms, which indicated evidences regarding criterion-related validity. The theoretical discussion was present based on the neuropsychological model of hot and cool aspects of executive function.

Keywords: Impulsivity, dimensionality, BIS-11.
\end{abstract}

\begin{abstract}
Resumo
O objetivo principal desse estudo foi investigar as características psicométricas da Barratt Impulsiveness Scale (BIS-11). A análise do conteúdo dos itens foi realizada por sete doutorandos. A amostra foi composta por 897 estudantes submetidos a BIS-11 e a medidas de autorrelato sobre a presença de sintomas de Transtorno Mental Comum e TDAH, uso de álcool e tabagismo. A idade média foi de $27,32(D P=8,69)$ anos, $56 \%$ eram mulheres e 52\% tinham educação superior incompleta. Análises de conteúdo e fatorial indicaram que a impulsividade é representada mais adequadamente por dois fatores denominados dificuldade de planejamento e controle inibitório. Concordância teste-reteste indicou que os escores se mantiveram estáveis após sete meses. Adicionalmente, os escores da BIS-11 discriminaram os indivíduos em termos de tabagismo e sintomas psicopatológicos, indicando evidências de validade de critério. A discussão dos resultados foi baseada no modelo neuropsicológico dos componentes quentes e frios das funções executivas.

Palavras-chave: Impulsividade, dimensionalidade, BIS-11.
\end{abstract}

At some point in life, most people have already engaged themselves in some sort of impulsive acts, such as taking too many drinks or making an non-planned purchase, talking about a topic that would be inappropriate to a certain moment and having racing thoughts instead of concentrating while solving problems. These actions are part of a fundamental dimension of personality, namely impulsivity. Impulsivity could be broadly defined as a behavior without

Mailing address: Departamento de Psicologia, Universidade Federal de Minas Gerais, Faculdade de Filosofia e Ciências Humanas, Laboratório de Avaliação das Diferenças Individuais, sala 4052, Av. Antônio Carlos, 6.627, Cidade Universitária, Pampulha, Belo Horizonte, MG, Brasil 31270-901.E-mail: alinagomide@gmail.com, mlmteodoro@hotmail.com,malloy.diniz@gmail.com and correa@task.com.br adequate thought or conscious judgment a tendency to act toward rapidly and in an unplanned manner, with less forethought about the future consequences (Moeller, Barratt, Dougherty, Schmitz, \& Swann, 2001). However, there are several definitions in the literature, which suggests that the construct possible comprises different components, based on different psychological and biological underlying mechanisms (Evenden, 1999).

A number of studies have investigated the structure of the impulsivity trait based on factor analyses from the set of responses to self-report scales. With regard to the taxonometric models, these exploratory factorial studies have already identified three dimensions, labeled non-planning, disinhibition and thrill-seeking (Flory et al., 2006), four impulsivity factors (impulsiveness, deliberation, selfdiscipline and excitement seeking; Whiteside \& Lynam, 2001) as well as seven components (prepared/careful, im- 
Vasconcelos, A. G., Teodoro, M. L. M., Malloy-Diniz, L. \& Correa, H. (2015). Impulsivity Components Measured by the Brazilian Version of the Barratt Impulsiveness Scale (BIS-11).

petuous, divertible, thrill and risk seeking, happy-go-lucky, impatiently pleasure seeking and reserved) which can be grouped into two principal components (unprepared/ spontaneous and sensation seeking; Kirby \& Finch, 2010).

It is noteworthy that these studies failed to establish a common framework among self-reported impulsivity items and they contributed to emphasize the multidimensionality nature of impulsivity. Emotional and cognitive executive function processes could be related to deliberating and controlling individual's responses (Bechara, 2004). This intersection gives a misleading indication that impulsivity refers to conceptually separable components when, in fact, they are interdependent. Hot-cool neuropsychological model of executive function could be useful to explain the multidimensionality of this construct (Zelazo \& Cunningham, 2007). For example, cool aspects are specialized in decontextualized problems, complex spatiotemporal tasks, declarative knowledge, episodic representation and abstract aspects of a certain situation which requires controlled and directed behavior. The hot EF aspects are particularly prominent when people care indeed about situations they are attempting to face because it involves motivational and affective aspects (Happaney, Zelazo, \& Stuss, 2004).

One of the best established self-report measure of impulsivity is the Barratt Impulsiveness Scale (BIS; Stanford et al., 2009). This instrument has been translated to many languages and it has influenced the conceptualization of impulsiveness in the literature. The current scale version is namely BIS-11 and was validated by Patton, Stanford and Barratt (1995). The authors identified three second-order factors: (a) motor impulsiveness (acting without inhibition of prepotent or ongoing responses), (b) attention impulsiveness (lack of focus on the ongoing task and comprised of two first-order factors, attention and cognitive instability), (c) nonplanning impulsiveness (characterized as an orientation to the present or lacking of future; Patton et al., 1995).

Stanford and colleagues (2009) demonstrated adequate psychometric proprieties for BIS-11 ( $N=1577$ adults), but only mentioned evidences about the adequacy of original factor structure. Recently, a review study has summarized the psychometric proprieties evidences of BIS-11 from 24 samples (Vasconcelos, Malloy-Diniz, \& Correa, 2012). The instrument showed good internal consistency for total score and criterion-related validity evidence. Yet, most of the studies were conducted in a wide range of cultures using adapted versions of the BIS-11 and they were unable to obtain the original 3-factor model or authors reported only two factors (Vasconcelos et al., 2012). These findings did not support the hypothesis that BIS-11 assesses the three impulsivity components originally supposed by Patton et al. (1995).

Theoretical and empirical evidences to support the interpretations of test scores are fundamental to indicate the degree scores captures important aspects of the construct and its relevance to the proposed use (American Educational Research Association [AERA], American
Psychological Association [APA], \& National Council on Measurement in Education [NCME], 1999). Thus, it appears necessary to join gather evidences regarding which impulsivity dimensions are being evaluated by BIS11 , due to the fact that this instrument is one of the most used impulsivity self-report measure in clinical as well as in research contexts (Stanford et al., 2009). Recently, an adult version was adapted by Malloy-Diniz, et al. (2010) to Brazilian context and it still needs to be validated. Diemen, Szobot, Kessler, and Pechansky (2007) had already adapted the Portuguese translation of BIS-11-A. However, BIS-11-A is a preliminary version of BIS-11 developed by Patton and colleagues (1995).

Our research question was convergent with validity arguments (see AERA et al., 1999; Urbina, 2007) and the main purpose of this study was to investigate psychometric properties of Brazilian version of BIS-11 using content, exploratory and confirmatory factorial analysis. Additionally, we aimed to investigate other BIS-11 psychometrics proprieties such as reliability, test-retest and the relationship between impulsive dimensions and demographic/ health variables (socioeconomic indicators, alcohol use, tobacco use and minor mental disorder symptoms). We hypothesized that impulsivity is a multidimensional construct in accordance with Barratt, however, the model with two separable impulsivity dimensions should provide the best fit to the data, according to preliminary analyses published elsewhere (see Ireland \& Archer, 2008; Vasconcelos et al., 2012).

\section{Method}

This study was part of an ongoing research entitled "Psychometric properties of the version of Barratt Impulsiveness Scale, version 11 (BIS-11) for Brazilian adults" approved by the Ethical Committee of the Universidade Federal de Minas Gerais in 2010. All individuals agreed to participate voluntarily and signed an Informed Consent Form.

The study was composed by two stages: Content items analyses, and Empirical analyses: (a) Pilot Exploratory Study and (b) Confirmatory Study and psychometric analysis. Each of these stages had specific samples and methodological issues, as presented below.

\section{Content Items Analyses}

Participants. Content item analysis was conducted by 7 doctoral students chosen due to their experience in personality and neuropsychological research.

Producers. Content items analyses were made using theoretical evidences based on impulsivity conceptual frameworks. The judges received two protocols composed by (a) constitutive definitions of the impulsivity domain, (b) two-input table where the rows contained BIS-11 items and the columns contained the impulsivity components. Judges had to analyze each item and identify which specific impulsivity domain it best represents. In the first protocol, 
impulsivity components were defined according to the empirical model proposed by Patton et al. (1995): motor impulsiveness, attention impulsiveness and nonplanning impulsiveness.

In the second protocol, two impulsivity dimensions were shown, non-planning (Bechara, 2004) and inhibitory control (Nigg, 2000). These dimensions were chosen because we could identify them in most of the BIS-11 psychometric studies (Vasconcelos et al., 2012). The appropriateness of an item to an alleged domain was established only if at least four judges (57.14\%) had recommended it.

\section{Empirical Analyses}

Participants. A convenience sample of 897 students from technical, graduates and undergraduate courses was submitted to BIS-11. Exclusion criteria were: age below 18 years old or above 62 years old, illiteracy and reported neurological disorders.

From this total sample, 175 individuals participated in a pilot preliminary study. Mean age was $27.32(S D=8.69)$ years old and $56 \%$ were female. Fifty two percent had incomplete college undergraduation. The answers of the other 722 participants were used to test confirmatory models and following psychometric analysis. Age ranged from 18 to 61 years old (mean=30.09; $S D=11.39$ ). Females constituted $56.1 \%$ of this sample. Forty two percent had incomplete college undergraduation and $64.6 \%$ were working and/or studying. Health variables indicated that $60.2 \%$ of individuals did not smoke and $52 \%$ did not consume alcohol. None of the participants reported having a psychiatric diagnosis made by a doctor. In a retest, 108 of these individuals accepted our invite and answered the BIS-11 again. The participants shown the mean age of $25.21(S D=3.76)$ years old and $82.6 \%$ were male. None of these participants reported cigarette use and $52.2 \%$ were alcohol consumers.

Instruments. All individuals completed the Portuguese adaptation of the BIS-11 (Malloy-Diniz et al., 2010). Furthermore, they filled measures of symptoms of Minor Mental Disorder (SRQ-20; Harding et al., 1980) and ADHD (ASRS-18, Adult Self Report Scale; Kessler et al., 2005) as well as reported whether they were alcohol users (CAGE scores; Amaral \& Malbergier, 2004) or cigarette smokers (FTND, Fagerström Test for Nicotine Dependence; Carmo \& Pueyo, 2002). Higher FTND scores indicate high level of nicotine dependence (Carmo \& Pueyo, 2002). The cutoff points for classification of nicotine dependence were $0-2$ points (very low severity), 3-4 points (low severity), 5 points (moderate severity), 6-7 points (high severity) and 8-10 points (very high severity).

\section{Producers.}

1. Pilot Exploratory Study. To demonstrate the internal structure of BIS-11, two exploratory factorial analyses (EFA) were performed with Promax factor rotation in a sample composed by 175 individuals (pilot preliminary study). The factorization was stopped at the extraction of 3 theoretically expected factors (Patton et al., 1995) and 2 factors according to the empirical evidences analyses (Vasconcelos et al., 2012).

2. Confirmatory Study and Psychometric Analysis. To investigate psychometric proprieties, a sample composed by 722 individuals was used. Confirmatory factor analyses (CFA) were used to compare items organization of 4 models: (a) Original 3-factor model, (b) Exploratory factor model, (c) Judges' 3-factor model and (d) Judges' 2-factor model. In order to identify the best fitting model, the following five goodness-of-fit statistics indicated the best fit to the data (Hair, Anderson, Tatham, \& Black, 2005): (a) Goodness-of-fit chisquare statistic: a non-significant $\chi^{2}$ statistic, (b) Root Mean Square Error of Approximation (RMSEA)£.05, (c) lower Akaike Information Criterion (AIC), (d) NonNormed Fit Index (NNFI) and Comparative Fit Index $(\mathrm{CFI})<.90,(\mathrm{e})\left(\chi^{2} / d f\right)$ and the standardized $\chi^{2}<5$. The correlations among latent variables would provide an estimation of the degree to which these factors were related to each other in the identification of a general impulsivity. Internal consistency was investigated by Cronbach's alpha.

Kolmogorov Smirnov test was used to test for normality of the scores distribution. Correlations coefficients were calculated for test-retest reliability. The retest was obtained from 108 participants, 7 months later, on a unique assessment section. To demonstrate the discrimination capacity of BIS-11 dimensions (e.g.: to discriminate individuals who would present minor mental/health problems), we used Student's $t$ test (normality distribution) or KruskalWallis test (non-normality distribution) and chi-square test. Finally, linear regression analyses were performed to determine the effects of health and sociodemographic variables on BIS-11 scores. When dependent variables did not show normal distribution, logarithmic transformations were applied. Analyses were performed with the SPSS version 19 and Lisrel version 8.8 programs. We used a level of $5 \%$ as the criterion for significance.

\section{Results}

The percentage of missing data ranged from 0 to $2.2 \%$ unanswered items. Item 16 ("I change jobs") had the highest percentage of non-responses (2.2\%), followed by the item 13 ("I make plans to keep me at work)" that shown $1.29 \%$ of non-responses.

\section{Content Items Analyses}

In the first protocol, which considered impulsivity components as proposed by Patton et al. (1995), the concordance between judges about items representing of one of the three domains (non-planning, motor, non-attention), or none of them, ranged from $28.57 \%$ to $100 \%$. Twenty items $(1,3,5,7-11,13,14,16,17,19-22,25,26,28,30)$ were associated with the same impulsivity dimension according to at least 5 judges $(71.43 \%)$. Other five items 
Vasconcelos, A. G., Teodoro, M. L. M., Malloy-Diniz, L. \& Correa, H. (2015). Impulsivity Components Measured by the Brazilian Version of the Barratt Impulsiveness Scale (BIS-11).

$(4,6,12,15$ and 22$)$ were more difficult to be matched to one of the three impulsivity components and only 4 judges matched them to the same dimension (57.14\%). The remaining items (27 and 29) were associated with the same dimension for at least 3 judges (42.86\%). The item 23 was hard to be associated to one proposed impulsivity dimension and the concordance between judges reached only $28.57 \%$. There was no agreement about which dimension item 18 represented. Finally, there was a moderate agreement between judges that items 15 and 29 did not represent any of the impulsivity dimensions proposed.

Considering the second protocol, the concordance between judges about items representing one of the two possible domains (planning or control inhibition), or none of them, ranged from $42.86 \%$ to $100 \%$. Twenty one items $(1,2,4,6-14,17,19,22,23,25-28,30)$ were associated with the same impulsivity dimension according to at least 5 judges $(71.43 \%)$. The remaining nine items $(3,16,18$, $20,21,24,29)$ were associated with the same dimension according to at least 4 judges (57.14\%). Item 5 was matched to inhibitory control based on the accordance between 3 judges $(42.86 \%)$. Finally, there was a moderate concordance between judges that items 15 and 29 did not represent any of the impulsivity dimensions proposed.

Evidence based on content items analysis indicated that there was a higher concordance of items organization between judges when considering two dimensions, namely inhibitory control and non-planning. The items organization based on content analysis is shown in Table 1.

\section{Empirical Analyses}

Exploratory Study ( $n=178)$. The KMO measure and Bartlett's test of sphericity indicated sampling adequacy to EFA (KMO $=.90$, statistic Bartlett's test of sphericity $=2625.24 ; d f=435 ; p<.001)$. All items presented communalities ranging from .3 to .65 , which indicated moderately satisfactory correlation with the factors (see Table 1; Hair et al., 2005).

The three-factor model explained $45.29 \%$ of the total variance of scores. Nonetheless, the factorial structure failed to replicate the original items organization. Most items loaded similarly on multiple components and none of them loaded exclusively on the third factor. It was not possible to label these factors in the same way as proposed by Patton and colleagues (1995).

The two-factor model was retained using the same EFA procedures. The results indicated that the factors explained $42 \%$ of scores' total variance. The factor loadings are presented in Table 1. Based on content analysis, we labeled the first dimension as inhibitory control given that it included motor impulsivity and lack of attention items from original scales. The second dimension was labeled as non-planning impulsivity. BIS-11 items organization based on Content Analysis and EFA are presented in Table 1.

When both 2-factor models were compared, five differences concerning the items organization could be observed. Based on content analysis, items 8, 9 and 20 were associ- ated with the inhibitory control dimension as well as the items 4 and 27 were linked to the non-planning dimension. The opposite pattern of association was observed on the exploratory model.

Confirmatory Study and Psychometric Analyses $(n=722)$. In order to test how well the original structure, the exploratory one and the structure obtained based on the judges' analysis CFA with maximum likelihood method were performed. Results are presented in Table 2.

The original and judges' 3 -factor models were not within the acceptable range $\left(\chi^{2} / d f\right.$ close to 5 ; RMSEA $=.80$; higher AIC). The other models presented satisfactory results in all three goodness of fit indexes and were in the acceptable range $\left(\chi^{2} / d f\right.$ nearest $<5$; RMSEA $\left.<.70 ; \mathrm{CFI}=.90\right)$. Moreover, AIC presented a best fit for the 2-factor model than for the other factor models. All goodness-of-fit indexes indicated that the two second-order factor models (obtained from EFA and content analysis) were the best fitting models.

Important validity evidence was obtained from content item analysis and it was used to justify our choice between the 2-factor models available. According AERA et al. (1999), content analysis could be more consistent about item representativeness. Furthermore, 2-factor model obtained from judge's analysis was chosen to be used in the following analyses. The final two dimensions assessed by BIS-11 were: (a) Factor 1: non-planning impulsiveness (NP) which included difficulty in delaying long-term gratification and difficulty in making choices after careful analysis of the possible consequences (Bechara, 2004; Evenden, 1999; Patton et al., 1995). (b) Factor 2: inhibitory control (IC) which included the ability to inhibit a dominant prepotent, automatic and/or in progress responses that are ineffective as well as competitive responses (interference control) during the interval between response inhibition and the new response (Nigg, 2000). According to CFA results, the association between the final 2 factors was .72 and that justifies forming one overall total score scale. To keep the scores as similar as possible to the original ones, total score was calculated with items 15 and 29 as part of the scale.

\section{Descriptive Statistics for BIS-11 Dimensions}

The descriptive statistics and Cronbach's alpha for the BIS- 11 and ASRS- 18 were calculated. On the one hand, descriptive scores for BIS-11 dimensions were: Inhibitory control (mean $=39.26, S D=8.56, a=.83$ ), Nonplanning (mean=17.88, $S D=4.16, a=.66$ ) and Total score (mean=57.14, $S D=11.21, a=.66$ ). On the other hand, descriptive statistics for ASRS-18 were: Attentional symptoms (mean $=13.89, S D=5.50, a=.85$ ) and Hyperactivity symptoms (mean $=12.80, S D=5.23, a=.88$ ).

Moreover, thirty-two percent of individuals reported minor mental disorders ( $N=143 / 447$ respondents); $32.5 \%$ ( $N=235 / 670$ respondents) were smokers, however, nicotine dependence severity was very low or low in $53.5 \%$ of participants, mild in $16.5 \%$, high or very high in $30 \%$, 
Psicologia: Reflexão e Crítica, 28(1), 96-105.

Table 1

BIS-11 Items Organization based on Content and EFA Analysis

\begin{tabular}{|c|c|c|c|}
\hline \multirow{2}{*}{ Abbreviated Items } & \multirow{2}{*}{ Content analysis } & \multicolumn{2}{|c|}{ Factors loading EFA } \\
\hline & & Factor I: IC & Factor II: NP \\
\hline 1. Plan tasks carefully. & NP & -.13 & .72 \\
\hline 2. Do things without thinking. & IC & .80 & -.06 \\
\hline 3. Make-up my mind quickly. & IC & .56 & -.26 \\
\hline 4. Am happy-go-lucky. & NP & .63 & -.12 \\
\hline 5. Don’t "pay attention". & $\mathrm{IC}$ & .72 & -.20 \\
\hline 6. Have "racing" thoughts. & IC & .65 & -.22 \\
\hline 7. Plan trips well ahead of time. & NP & -.24 & .64 \\
\hline 8. Am self controlled. & $\mathrm{IC}$ & -.11 & .68 \\
\hline 9. Concentrate easily. & IC & -.14 & .67 \\
\hline 10. Save regularly. & $\mathrm{NP}$ & -.12 & .59 \\
\hline 11. "Squirm" at plays. & IC & .68 & -.24 \\
\hline 12. Am a careful thinker. & NP & -.22 & .75 \\
\hline 13. Plan for job security. & NP & -.09 & .67 \\
\hline 14. Say things without thinking. & IC & .75 & -.02 \\
\hline 15. Think about complex problems. & $\mathrm{x}$ & -.44 & .56 \\
\hline 16. Change jobs. & IC & .56 & -.33 \\
\hline 17. Act "on impulse". & IC & .80 & -.12 \\
\hline 18. Get bored solving thought problems. & IC & .67 & -.20 \\
\hline 19. Act on the spur of the moment. & IC & .78 & -.10 \\
\hline 20. Am a steady thinker. & $\mathrm{IC}$ & -.17 & .72 \\
\hline 21. Change residences. & IC & .62 & -.33 \\
\hline 22. Buy things on impulse. & IC & .70 & -.24 \\
\hline 23. Think about one thing at a time. & IC & .52 & -.36 \\
\hline 24. Change hobbies. & IC & .70 & -.26 \\
\hline 25. Spend more than I earn. & IC & .64 & -.09 \\
\hline 26. Have extraneous thoughts. & IC & .61 & -.23 \\
\hline 27. Interested in the present. & NP & .53 & -.19 \\
\hline 28. Am restless at theater. & IC & .67 & .00 \\
\hline 29. Like puzzles. & $\mathrm{x}$ & -.29 & .55 \\
\hline 30. Am future oriented. & NP & -.15 & .67 \\
\hline
\end{tabular}

Note. The highest loading or correlation for each scale is in bold. NP =Non-planning; IC=Inhibitory control; $\mathrm{x}=$ item did not represent any impulsivity dimension. 
Vasconcelos, A. G., Teodoro, M. L. M., Malloy-Diniz, L. \& Correa, H. (2015). Impulsivity Components Measured by the Brazilian Version of the Barratt Impulsiveness Scale (BIS-11).

Table 2

BIS-11 Confirmatory Factor Analysis Models

\begin{tabular}{lcccccccc}
\hline \multicolumn{1}{c}{ Models } & $\chi^{2}$ & $d f$ & $\chi^{2} / d f$ & RMSEA & AIC & NNFI & CFI & GFI \\
\hline Original 3-factor & $2209.68^{*}$ & 402 & 5.50 & .079 & 2335.68 & .87 & .88 & .83 \\
Exploratory 2-factor & $\mathbf{1 4 8 8 . 4 4 *}$ & $\mathbf{3 7 6}$ & $\mathbf{3 . 9 6}$ & $\mathbf{. 0 6 4}$ & $\mathbf{1 6 0 6 . 4 4}$ & $\mathbf{. 9 0}$ & $\mathbf{. 9 1}$ & $\mathbf{. 8 8}$ \\
Judges' 3-factor & $1640.69^{*}$ & 320 & 5.13 & .075 & 1756.69 & .88 & .89 & .86 \\
Judges' 2-factor & $\mathbf{1 5 9 4 . 4 8 *}^{*}$ & $\mathbf{3 4 9}$ & $\mathbf{4 . 5 7}$ & $\mathbf{. 0 7 0}$ & $\mathbf{1 7 0 8 . 4 8}$ & $\mathbf{. 8 9}$ & $\mathbf{. 9 0}$ & $\mathbf{. 8 6}$ \\
\hline
\end{tabular}

Note. Bold numbers represent an acceptable fit model; RMSEA=root mean square error of approximation; AIC=Akaike information criterion; $\mathrm{NNFI}=$ non-normed fit index; $\mathrm{CFI}=$ comparative fit index; GFI=Goodness of fit index. Items 15 and 29 were not included on the confirmatory models.

$* p<.0001$.

of participants. Finally, $48 \%(N=254 / 529$ respondents $)$ reported alcohol use and $13.6 \%(N=35 / 254$ respondents $)$ reported the presence of alcohol abuse (the cutoff point was considered as being above two points in CAGE).

The internal consistency coefficients ranged from moderate to good. Participants' performances on BIS-11 scores did not show a normal distribution $(1.89<\mathrm{Z}<2.63 ; p<.001)$ as well as ASRS-18, FTND and CAGE $(1.33<Z<5.11$; $p<.05)$. Using correlation coefficient, associations between test and retest were for IC $(\mathrm{rho}=.65, p<.001)$, NP and total scores $(\mathrm{rho}=.72, p<.001)$. Correlations results indicated moderate stability over the 7 -months period.

Influence of Demographic Variables on BIS-11 Scores

There were no differences between sex $(58640<U<$ $61346, p>.05)$ and marital status $(1.23<$ chi-square $<3$, $p>.05)$ on BIS-11 scores. The correlations between the scores and the subjects' ages were non-significant for total score and IC dimension $(-.07<$ rho $<-.01)$ and significantly low for NP dimension ( $\mathrm{rho}=-.10, p=.01$ ).

We found significant correlations between years of schooling and BIS-11 scores (total: rho=-.30; IC: rho=.30 and NP: rho=-.20; $p<.001)$. In this study, individuals with lower educational level showed higher scores (Total: mean=59.20; $S D=11.92 ; \mathrm{IC}:$ mean $=40.24 ; S D=9.14$; NP: mean $=18.97 ; S D=4.33$ ) than graduate and undergraduates participants (Total: mean $=55.53 ; S D=10.45$; IC: mean $=38.47 ; S D=8.06 ; \mathrm{NP}:$ mean $=17.00 ; S D=3.80$; $U>45678 ; p<.001)$.

\section{Other Validity Evidences}

All items demonstrated an item-scale correlation with their impulsivity dimension higher than with another one. Some items had similar associations with both dimensions (e.g.: 8, 20, 21). Convergent with the results of the content analysis, items 15 and 29 demonstrated the lowest correlations with both impulsivity dimensions. Therefore, they were not appropriate representatives of any impulsivity dimension proposed.

To explore the concurrent validity evidences, associations between BIS-11 scales and ADHD symptoms, cigarette smoking and alcohol use were investigated by simple regression analysis. Models were adjusted considering the years of schooling effects using multiple regression analysis. The coefficients of these regression models are listed in Table 3.

Table 3

Association between BIS-11 Scores and Health Variables

\begin{tabular}{|c|c|c|c|}
\hline \multirow{3}{*}{ Independent Variables } & \multicolumn{3}{|c|}{ Dependent variables } \\
\hline & \multicolumn{3}{|c|}{ Standardized coefficients } \\
\hline & $\mathrm{IC}$ & NP & Total \\
\hline Attentional symptoms ${ }^{\text {a }}$ & $.65^{* *}$ & $.32 * *$ & $.62 * *$ \\
\hline Hyperativity symptoms ${ }^{a}$ & $.64 * *$ & $.26 * *$ & $.59 * *$ \\
\hline Cigarette Smoker & $.21 * *$ & $.31 * *$ & $.27 * *$ \\
\hline Alcohol use & $.11 *$ & $.18^{*}$ & $.18 *$ \\
\hline $\begin{array}{l}\text { Note. NP =Non-planning; IC=Inhibito } \\
\text { aLogarithmic transformations were us } \\
{ }^{*} p<.05 ; * * p<.001 \text {. }\end{array}$ & & & \\
\hline
\end{tabular}


As shown in Table 3, BIS-11 scores correlated significantly with ADHD symptoms and cigarette smoking. They were not substantially influenced by subjects' educational level since the standardized coefficients showed similar values on multiple models controlling for the effect of education. The exception was the presence of alcohol use which showed no association with NP score when removing the effects of education. Smokers and alcohol users showed higher BIS-11 scores.

Considering the mental screening instruments as the cut off criterion, individuals that reported the presence of minor mental symptoms tended to be more impulsive on three scores $(U>11520, p<.001)$. Additionally, individuals that reported presence of ADHD symptoms tended to be more impulsive based on Kruskal-Wallis tests, $\chi^{2}$ (2) $>7.84 ; p<.05$.

\section{Discussion}

This study identified that a two-factor model fitted BIS-11 the best, based on empirical and theoretical analyses. The internal consistency was satisfactory and similar to the values previously reported for the BIS-11 in adult subjects (Stanford et al., 2009; Vasconcelos et al., 2012). Test retest agreement tended to produce similar score patterns seven months after the first evaluation and it also presented adequate correlations coefficients between the two periods. Eeducational level and socio-economic status influenced all BIS-11 scores. Additionally, BIS-11 scores significantly discriminated subjects in terms of presence of cigarette smoking and psychopathology symptoms, which indicated evidences regarding criterion-related validity.

As Vasconcelos et al. (2012) suggested, the original BIS-11 factor structure has not been supported by most of the factor analysis studies conducted in a wide range of cultures. In the present study, we could observe that third dimension was badly defined based on expert judgments as well as on the EFA, with weighty items loading in different factors. From the theoretical point of view, content analysis of the items organization indicated that 2 -factor models were more coherent in a theoretical point of view. This result was convergent with EFA and itemscale correlations.

CFA demonstrated that both bi-dimensional models based on judges and EFA fitted BIS-11 well. Following AERA et al. (1999) recommendation, we have chosen content analyses because original BIS-11 scales have already been developed on empirical factor analysis. Thus, expert judgments were used to assess the relative construct that a certain item represented and also to evaluate how appropriate the item would be in relation to the impulsivity dimension it originally represented

Factors names proposed by Barratt were not maintained, once dimensions' content did not exactly fit to the original factors. Taken at content validity, we labeled the observed dimensions as "inhibitory control" and "nonplanning" impulsiveness. The first factor combined motor and attention original dimensions and was convergent with a broad type of inhibitory behavior which is relevant to both motor control and working memory functions. Inhibitory control could be defined as the ability to suppress internal stimuli that may interfere with the current operations of working memory by keeping unwanted or non-relevant thoughts out of mind and/or to inhibit a dominant or prepotent primary response (Barkley, 1997). The observed structured differed from Barratt's aim to measure attention and motor constructs separately (Patton et al., 1995), but it corroborates Patton's conclusion that basic cognitive processes might underlie the personality trait of impulsiveness (Hartmann \& Rief, 2011; Patton, et al., 1995). The second factor included non-planning items and referred to the ability to delay gratification and evaluate future outcomes of a planned action (Evenden, 1999).

This result is especially significant for three reasons: Firstly, supporting this BIS-11 second-order factors, Congdon and Canli (2008) suggested several empirical studies that provided agreeable evidence to the hypothesis that impulsivity is composed for at least two dimensions: (a) disinhibition and (b) decision making, markedly characterized by a diminished capacity to plan a delay. This suggests that these factors could be proposed as some sort of general aspects of impulsivity, identifiable across the studies. Secondly, although Patton et al.'s (1995) factor structure could not be perfectly replicated, findings in other 2-factor models studies were quite similar (Vasconcelos et al., 2012). Additionally, joining uniting Hartmann and Rief (2011)'s factors 1 (mainly composed by cognitive and motor items) and 3 (consisted of four motor items), led to the final structure General and Non-planning Impulsiveness found in the present study. One possible reason for this result is the fact that the differentiation between motor and attentional impulsiveness aspects could not be easily done in terms of behaviour operationalization, especially when using a self-report questionnaire.

Thirdly, from the theoretical rationale interpretation, BIS-11 dimensions could be considered as behavioral representations of cognitive and affective aspects of executive functions (EF). The ability to control a behavior depends on interactions between distinct elements from "hot" motivationally significant aspects of $\mathrm{EF}$ and the more disinterested "cool" aspects (Zelazo \& Müller, 2002). On the one hand, BIS-11 inhibitory control items could be understood as basic behaviors especially influenced by cool EF process. On the other hand, BIS-11 Non-planning items could be seen as behavior representations of hot EF processes. As an example, planning the future requires many cognitive abilities influenced by emotional considerations that allow individuals to have a long-term realistic view of a situation, to process the reward in unstructured situations, as well as to demonstrate appropriate social interactions with friends and family (Hernadez, Denburg, \& Tranel, 2009; Stuss \& Levine, 2002).

Anatomical and neuropsychological distinctions associated to hot and cool EF were identified based on empirical 
Vasconcelos, A. G., Teodoro, M. L. M., Malloy-Diniz, L. \& Correa, H. (2015). Impulsivity Components Measured by the Brazilian Version of the Barratt Impulsiveness Scale (BIS-11).

research as could be seen on Bechara's (2004), Stuss and Levine's (2002) and Zelazo and Cunningham's (2007) studies. The cool cognitive system was consistent with the dorsolateral prefrontal cortex (DL-PFC), a circuit that plays an important role in the integration of sensory and mnemonic information and the regulation of intellectual function and action (Fuster, 2005; Stuss \& Levine, 2002). In contrast, the hot system was mediated by ventromedial prefrontal cortex (VM-PFC) and its subcortical regions are responsible for managing emotionally relevant information which underlies self-control, planning and decision making (Fuster, 2005; Stuss \& Levine, 2002).

One could argue that planning ability, as measured in this study, (hot process as an example) is influenced by analytic situational aspects as well as inhibitory control (such as cool processes) and requires emotional regulation of stressful aspects of daily life. In fact, behavior regulation involves both hot EF (control processes centered on reward representations) and cool EF (higher-order processing of more abstract information; Zelazo \& Cunningham, 2007). Furthermore, although conceptually separable, the emotional and cognitive EF are related to deliberating and controlling individual's responses, since the influence of emotions on cognitive process could not be ignored (Bechara, 2004; Evenden, 1999). DL-PFC and VM-PFC are parts of an interactive functional system and they typically work together (Happaney et al., 2004).

Our study provides supporting evidence to this point of view by showing that BIS-11 dimensions (inhibitory control and non-planning) were moderately associated to each other. Therefore, they likely share cognitive and emotional aspects. Additionally, the interconnections between hot and cool EF have important implications for the interpretation of the instruments proposed to assess them. We hypothesized that the overlapping between these components could influence the degree to which BIS- 11 items failed to capture pure aspects of the dimensions originally proposed and interfere in the identification of a stable empirical internal structure across different samples. One item could elicit broad impulsiveness aspects and this makes it difficult to recognize which component one item represents the best. Inhibitory control tends to be influenced by affective reactions and this could partially explain why items 8,9 and 20 loaded on BIS- 11 hot dimension although the content analysis organized them on cool dimension (inhibitory control). Individuals' abilities to concentrate, to be self-controlled and steady thinkers seem likely to be influenced by motivational and emotional states (hot process) besides they were being basically inhibitory control behaviors. Zelazo and Cunningham (2007) proposed considering hot-cool $\mathrm{EF}$ as a continuum that corresponds to the motivational significance of the to-be-solved problem and to its reflection degree. So it is necessary to adopt a much more theoretical approach when trying to understand the meaning of impulsiveness scores (AERA et al., 1999). Our validation study carefully investigates possible distortions in the empirical structure that arises from inadequate construct representation. Empirical and theoretical BIS-11 studies are important to better understanding the meaning of these scores.

The retest reliabilities of BIS-11 dimensions were acceptable and this indicated that BIS-11 was more sensitive to stable aspects of impulsivity than to transient changes. Finally, the concurrent validity evidence of BIS-11 was satisfactory when considering ADHD symptoms and similar to the concurrent validity evidence provided by Stanford et al. (2009). As expected, BIS-11 IC factor showed higher correlation with attention and hyperactive symptoms than BIS-11 NP dimension. The former is a more complex cognitive process that might not be impaired on the clinical sample with attention and hyperactivity self-reported difficulties in the same way as they would present deficits in IC. These results were convergent with Barkley's hypothesis (1997) which suggested that the essential impairment in subjects with ADHD involves response inhibition that leads to the decreased control of motor behavior and cognitive interference. These abilities are likely associated to DL-PFC (Bechara \& Van Der Linden, 2005). Additionally, cognitive impulsivity, which includes planning difficulties, tended to be associated with VM-PFC (Bechara, Tranel, \& Damasio, 2000) which could show secondary impairments due to the decrease of response inhibition.

The instrument also demonstrated good criterionrelated validity evidences. Individuals reported higher BIS-11 scores in the presence of psychiatric, cigarette use and alcohol abuse. Neuropsychological deficits were also observed on substances users, such as low motivation, poor impulsivity control, working memory deficits and poor planning ability because they ignored the future consequences of their acts (Bechara \& Martin, 2004). These results can be thought of as evidences that BIS-11 could be used to screen subjects with maladaptive styles of everyday life or who are at risk of substance abuse (Vasconcelos et al., 2012).

Different from what Patton et al. (1995) and Stanford et al. (2009) identified, the influence of socioeconomic status, educational level and age contributed to BIS-11 scores as previously shown elsewhere (Vasconcelos et al., 2012). Older individuals or individuals with higher educational level tend to be more self-controlled and better at planning. These evidences emphasized the importance of investigating the influence of demographic variables on the interpretation of scale scores.

Our factor analyses results could contribute to the improvement of the characterization of impulsivity components based on Barratt proposal, for the reason that the majority of research using the BIS-11 reports only the total score. Moreover, we also supposed ADHD symptoms, cigarette smoking and alcohol abuse were characterized more impulsive individuals (concurrent validity evidences) controlling the demographic effects.

The present study was the first, to our knowledge, to use the hot-cool neuropsychological model of executive 
function to explain BIS-11 dimensions. Noteworthy, this study discussed how the instability in the factor structure across different studies could be more related to problems with the underlying construct than to methodological issues or culture influences (Fossati, Barratt, Acquarini, \& Di Ceglie, 2002). There has been accumulating evidence that impulsivity is a multidimensional factor and its heterogeneous manifestations could influence the degree to which scores purely capture specific aspects of the construct. Important evidences come from the test content analysis, which provided the theoretical rationale to better understand the BIS-11 dimensions.

However, the current study presented a few methodological limitations and results should be interpreted with caution. First, the sample was composed by students and they are not representative of the general population. Heterogeneity samples are necessary to calculate normative parameters to scores interpretations. Moreover, this sample bias could explain the low association between BIS-11 factors and specific items, for example, item 16 (Change jobs).

Second, retest study was performed with a homogeneous group considering demographic and health variables. Thus, it could be argued that this characteristic contributed to the stability of the scores. Further studies are needed to confirm the bifactorial structure. It is important to emphasize that differences can be accounted for connotation specificities from the original and the Portuguese versions, despite the severe processes of translation, back-translation and the final Brazilian version. These differences could bias the internal structure organization.

Additionally, only a self-reported measure was used and some people could have difficulties to provide reliable information about their own behaviors and health history. Finally, BIS-11 dimensions were associated with hot-cool executive components following a theoretical rationale analysis. The understanding of neuropsychological aspects of BIS-11 components could be enhanced if further studies assessed convergent empirical evidences to assure that self-report scores are representatives of both functional and neural aspects involved in inhibitory control and planning abilities. Finally, besides smokers and alcohol users showed higher BIS-11 scores, partial scores showed weak associations with nicotine dependence symptoms and alcohol use. The hypothesis is that the majority of the participants reported low to middle nicotine dependence and alcohol use according the instruments' cutoff point. Future studies should assess impulsivity in addictive samples using BIS-11 adapted version for Brazilian adults.

In summary, although there are limitations, BIS-11 showed adequate psychometric proprieties based on a great Brazilian adult sample. Our scientific efforts have been benefited from psychometric and neuropsychological approaches to reach objective and theoretical frameworks that underlay individual performance on an impulsivity self-reported questionnaire. We continue the psychometric studies using BIS-11 records from heterogeneous sample composed by healthy individuals and mixed clinical groups with minimum introspective capacity. BIS-11 future researches should focus on neuroimaging studies to identified neuroanatomical correlates and on the administration of neuropsychological tasks traditionally used to assess hot and cool aspects of EF, particularly in clinical samples. The incorporation of functional and anatomical measures would im rove executive functions assessment (Stuss \& Levine, 2002).

\section{References}

Amaral, R. A., \& Malbergier, A. (2004). Avaliação de instrumento de detecção de problemas relacionados ao uso do álcool (CAGE) entre trabalhadores da Prefeitura do Campis da Universidade de São Paulo (USP) - Campus Capital. Revista Brasileira de Psiquiatria, 26(3), 156-183. doi:10.1590/ S1516-44462004000300005

American Educational Research Association, American Psychological Association, \& National Council on Measurement in Education. (1999). Standards for educational and psychological testing. Washington, DC: American Educational Research Association.

Barkley, R. A. (1997). Behavioral inhibition, sustained attention and executive functions: Constructing a unifying theory of ADHD. Psychological Bulletin, 121, 65-94. doi:10.1037/0033-2909.121.1.65

Bechara, A. (2004). The role of emotion in decision-making: Evidence from neurological patients with orbitofrontal damage. Brain and Cognition, 55, 30-40. doi:10.1016/j. bandc.2003.04.0

Bechara, A., \& Martin, E. M. (2004). Impaired decision making related to working memory deficits in individuals with substance addictions. Neuropsychology, 18(1), 152-162. doi:10.1037/0894-4105.18.1.152

Bechara, A., Tranel, D., \& Damasio, H. (2000). Characterization of the decision-making deficit of patients with ventromedial prefrontal cortex lesions. Brain, 123, 2189-2202. doi:10.1093/ brain/123.11.2189

Bechara, A., \& Van Der Linden, M. (2005). Decision-making and impulse control after frontal lobe injuries. Current Opinion in Neurology, 18, 734-739. doi:10.1097/01. wco.0000194141.56429.3c

Carmo, J. T., \& Pueyo, A. A. (2002). A adaptação ao português do Fagerström Test for Nicotine Dependence (FTND) para avaliar a dependência e tolerância à nicotina em fumantes brasileiros. Revista Brasileira de Medicina, 59, 73-80.

Congdon, E., \& Canli, T. (2008). A neurogenetic approach to impulsivity. Journal of Personality, 76(6), 1447-1484. doi:10.1111/j.1467-6494.2008.00528.x

Diemen, L. V., Szobot, C. M., Kessler, F., \& Pechansky, F. (2007). Adaptation and construct validation of the Barratt Impulsiveness Scale (BIS 11) to Brazilian Portuguese for use in adolescents (Brief Report). Revista Brasileira de Psiquiatria, 29(2), 153-156. doi:10.1590/S1516-44462006005000020

Evenden, J. L. (1999). Varieties of impulsivity. Psychopharmacology, 146, 348-361.

Flory, J. D., Harvey, P. D., Mitropoulou, V., New, A. S., Silverman, J. M., Siever, L. J., \& Manuck, S. B. (2006). Dispositional impulsivity in normal and abnormal samples. Journal of Psychiatric Research, 40, 438-447. doi:10.1016/j. jpsychires.2006.01.008 
Vasconcelos, A. G., Teodoro, M. L. M., Malloy-Diniz, L. \& Correa, H. (2015). Impulsivity Components Measured by the Brazilian Version of the Barratt Impulsiveness Scale (BIS-11).

Fossati, A., Barratt, E. S., Acquarini, E., \& Di Ceglie, A. (2002). Psychometric properties of an adolescent version of the Barratt Impulsiveness Scale-11. Perceptual and Motor Skills, 95(2), 621-635. doi:10.2466/pms.2002.95.2.621

Fuster, J. (2005). The prefrontal cortex. London: Academic Press.

Hair, J., Anderson, R., Tatham, R., \& Black, W. (2005). Análise multivariada de dados. Porto Alegre, RS: Bookman.

Happaney, K., Zelazo, P. D., \& Stuss, D. T. (2004). Development of orbitofrontal function: Current themes and future directions. Brain and Cognition, 55, 1-10. doi:10.1016/j. bandc.2004.01.001

Harding, T. W., De Arango, M. V., Baltazar J., Climent, C. E., Ibrahim, H. H. A., Ladrido-Ignacio, L, ...Wig, N. N. (1980). Mental disorders in primary health care: A study of their frequency and diagnosis in four developing countries. Psychological Medicine, 10, 231-241. doi:10.1017/ S0033291700043993

Hartmann, A. S., \& Rief, W. (2011). Psychometric properties of the German version of the Barratt Impulsiveness Scale, Version 11 (BIS-11) for adolescents. Perceptual and Motor Skills, 112, 353-368. doi:10.2466/08.09.10.PMS.112.2.353-368

Hernadez, M., Denburg, N. L., \& Tranel, D. (2009). A neuropsychological perspective on the role of the prefrontal cortex in reward processing and decision-making. In J. C. Dreher \& L. Tremblay, Handbook of reward and decision making. New York: Elsevier.

Ireland, J. L., \& Archer, J. (2008). Impulsivity among adult prisoners: A confirmatory factor analysis study of the Barratt Impulsivity Scale. Personality and Individual Differences, 45(4), 286-292. doi:10.1016/j.paid.2008.04.012

Kessler, R. C., Adler, L., Ames, M., Demler, O., Faraone, S., Hiripi, E., ...Walters, E. E. (2005). The World Health Organization Adult ADHD Self-Report Scale (ASRS). Psychological Medicine, 35(2), 245-256. doi:10.1017/S0033291704002892

Kirby, K. N., \& Finch, J. C. (2010). The hierarchical structure of self-reported impulsivity. Personality and Individual Differences, 48, 704-713. doi:10.1016/j.paid.2010.01.019

Malloy-Diniz, L. F., Mattos, P., Leite, W. B., Abreu, N., Coutinho, G., Jardim, J., ...Fuentes, D. (2010). Tradução e adaptação cultural da Barratt Impulsiveness Scale (BIS-11) para aplicação em adultos brasileiros. Jornal Brasileiro de Psiquiatria, 59(2), 99-105. doi:10.1590/S0047-20852010000200004

Moeller, F. G., Barratt, E. S., Dougherty, D. M., Schmitz, J. M., \& Swann, A. (2001). Psychiatric aspects of impulsivity. The American Journal of Psychiatry, 158(11), 1783-1793. doi:10.1176/appi.ajp.158.11.1783

Nigg, J. T. (2000). On inhibition/disinhibition in developmental psychopathology: Views from cognitive and personality psychology and a working inhibition taxonomy. Psychological Bulletim, 126(2), 220. doi:10.1037/0033-2909.126.2.22

Patton, J. H., Stanford, M. S., \& Barratt, E. S. (1995). Factor structure of the Barratt Impulsiveness Scale. Journal of Clinical Psychology, 51(6), 768-774. doi:10.1002/10974679(199511)51:6<768::AID-JCLP2270510607>3.0.CO;2-1

Stanford, M. S., Mathias, C. W., Dougherty, D. M., Lake, S. L., Anderson, N. E., \& Patton J. M. (2009). Fifty years of the Barratt Impulsiveness Scale: An update and review. Personality and Individual Differences, 47(5), 385-395. doi:10.1016/j. paid.2009.04.008

Stuss, D. T., \& Levine, B. (2002). Adult clinical neuropsychology: Lessons from studies of the frontal lobes. Annual Review of Psychology, 53, 401-433. doi:10.1146/annurev. psych.53.100901.135220
Urbina, S. (2007). Fundamentos da testagem psicológica. Porto Alegre, RS: Artmed.

Vasconcelos, A. G., Malloy-Diniz, L., \& Correa, H. (2012). Systematic review of psychometric properties of Barratt Impulsiveness Scale - Version 11 (BIS-11). Clinical Neuropsychiatry - Journal of Treatment Evaluation, 9(2), 61-74.

Whiteside, S. P., \& Lynam, D. R. (2001). The Five Factor Model and impulsivity: Using a structural model of personality to understand impulsivity. Personality and Individual Differences, 30, 669-689. doi:10.1016/S0191-8869(00)00064-7

Zelazo, P. D., \& Cunningham, W. A. (2007). Executive function: Mechanisms underlying emotion regulation handbook of emotion regulation. In J. J. Gross (Ed.), Handbook of emotion regulation (pp. 135-158). New York: Guilford Press.

Zelazo, P. D., \& Müller, U. (2002). Executive function in typical and atypical development. In U. Goswami (Ed.), Handbook of childhood cognitive development (pp. 445-469). Oxford, UK: Blackwell.
Recebido: $31 / 07 / 2013$

$1^{a}$ revisão: 13/01/2014

Aceite final: 16/01/2014 